\title{
OPTIMAL POWER CONTROL LAW FOR EQUAL-RATE DS-CDMA NETWORKS GOVERNED BY A SUCCESSIVE SOFT INTERFERENCE CANCELLATION SCHEME
}

\author{
Francesc Molina, Josep Sala-Alvarez, Javier Villares and Francesc Rey
}

\author{
Signal Theory and Communications Department, Technical University of Catalonia (UPC) \\ E-mail: $\{$ francesc.molina, josep.sala, javier.villares, francesc.rey $\} @$ upc.edu
}

\begin{abstract}
This paper studies the throughput maximization of a dense multiple access network of low-rate subscribers that share the same practical Forward Error Correction (FEC) code and modulation scheme, and transmit to a central node that implements a Successive Soft Interference Cancellation (soft SIC) strategy in order to mitigate Multiple Access Interference (MAI). In the user-asymptotic case, we make use of Variational Calculus (VC) tools to derive, in terms of the Packet Error Rate (PER) of the shared encoder and the Residual Energy (RE) from imperfect cancellation, the optimum energy profile that maximizes the network spectral efficiency, when a sum power constraint at the SIC input is enforced. Comparative performance analyses using a representative encoder are carried out. Simulation results show the benefit of the adopted soft SIC scheme in front of other SIC strategies, obtaining relevant throughput gains under high traffic loads.
\end{abstract}

Index Terms- Successive Soft Interference Cancellation, Packet Error Rate, Residual Energy, Variational Calculus

\section{INTRODUCTION}

A wide scope of machine-type communication systems has spurred the development of more sophisticated medium access control and physical layer capabilities [1]. In particular, with the focus on massively populated satellite networks, the amount of MAI constitutes their major performance limitation. In that respect, as stated in [2], different satellite communication standards, such as DVB-RCS2 [3] and SMIM [4], have encountered the necessity of incorporating IC strategies to allow successful MAI mitigation [5]. Enhanced Spread Spectrum Aloha, a system employing coded DirectSequence (DS) Code-Division-Multiple-Access together with SIC at reception [6][7], has been proposed as a powerful solution with an affordable implementation complexity [8][9]. Previous works have addressed network throughput optimization in the large-user regime under specific hard SIC policies [10][11]. In the machine-to-machine context, characterized

Work supported by projects TEC2016-76409-C2-1-R (WINTER) and TEC2013-47020-C2-2-R (COMPASS): Ministerio de Economía, Industria y Competitividad. Acknowledgements due to the reviewers. by low/moderate payload sizes, it becomes important to optimize the spectral efficiency of those multiple access protocols that aim to reduce the user-level overhead while maintaining the receiver complexity.

We recover the scenario in [10], where a satellite operating as a relay between a large population network and a gateway station is envisaged. In that context, the first contribution of this paper is the hitherto unexplored VC-based optimization of the system's spectral efficiency when a soft SIC (rather than a hard SIC [10]) operates at the gateway. Specifically, we derive the optimum power control law of a massive spread-spectrum multiple access network under synchronous access when: (i) users share the same practical encoding and modulation scheme, associated with a known PER vs. SINR curve, as well as a known RE vs. SINR curve corresponding to the soft cancellation procedure; (ii) the satellite repeater sets a sum power constraint over the user population. With respect to (i), this work has obtained the true RE vs. SINR curve of the iterative FEC decoder, rather than utilized the non-informative uniform (SINR-independent) assumption in previous works. We evidence that the adopted soft SIC decoding policy and the associated VC-based optimization procedure outperform the previously used strategies [10][11].

Our second contribution is threefold. Firstly, we obtain (Eq. (11)) a theoretical expression for the parameter $t_{0}$ governing load regulation under high traffic loads. In this regime, both the first and the last user's SINR value remain fixed. Surprisingly, in terms of the SINR of the last user, the activation of load regulation depends only on the PER curve of the encoder. Secondly, for application in system optimization, an exact differential (Eq. (13)) is shown to express the increments (decrements) of the optimum per-user Packet Success Rate over SIC stages in terms of increments of an equivalent magnitude related to the per-user symbol energy and SINR. Finally, our last contribution comprises the characterization of spectral efficiency under load regulation. We show that the maximum network throughput is achieved exactly when load regulation starts to operate. This contrasts with previous works [11] considering a SIC operating in the noisedominated conditions and with user transmissions subject to a long-term per-user average energy constraint, where the network throughput does not collapse for increasing loads. 


\section{SYSTEM MODEL}

We consider a very large population of $1 \leq k \leq K$ low-rate users transmitting towards a satellite repeater, in which the user index is established in decreasing order of the user channel power gains $h[k]$. For an affordable theoretical analysis, we have evaluated the synchronous access mode implemented in some standards [4] and also analyzed in [10][11][12]. At the physical layer, the same coding and modulation scheme is considered for all users, in which symbol sequences $s_{k}[n]$ are generated. A DS symbol spreading model is adopted whereby sequences of spreading gain $N$ are chosen randomly as portions of a long pseudorandom code.

We consider the autonomous operation of the network devices. When channel time-variations are sufficiently low and channel reciprocity holds, users can select their transmitted symbol energy, $E_{\mathrm{x}}[k]$, as function of their individual channel gains as long as perfect estimations of $h[k]$ are available from a downlink pilot signal. Thus, power control is rationally justified. At the satellite repeater, to ensure linear amplification, the system optimization is carried out holding the operating point (OP) of the on-board power amplification chain

$$
\mathrm{OP} \triangleq N_{u} \cdot r_{c} \cdot(1+\beta)+\alpha \cdot \bar{E} \cdot r_{c} \cdot\left(G_{s} h_{d}\right)^{-1},
$$

with $N_{u}$ the uplink noise power spectral density, $\beta$ the rolloff factor of the shaping pulse, $\alpha \triangleq K / N$ the system's load, $G_{s}, h_{d}$ the satellite and common channel downlink power gains, respectively, and $\bar{E} \triangleq \frac{1}{K} \sum_{k} E_{s}[k]$ the over all users average symbol energy. For a fixed chip rate $r_{c}$ that matches the channel's coherence bandwidth, $\mathcal{C} \triangleq \alpha \bar{E}$ is held constant once the OP is set. At the gateway, users are received with symbol energies $E_{s}[k]=E_{\mathrm{x}}[k] h[k] G_{s} h_{d}$. In order to mitigate the incoming MAI, the receiver is set to perform a successive decoding strategy. When perfect amplitude estimation is available, users are sorted in decreasing order of their symbol energies $E_{s}[k]$ and sequentially demodulated, followed by a soft reconstruction, $\hat{s}_{k}[n]$, and cancelled from the input signal before proceeding to the subsequent user. At the gateway, we consider the decoder's PER function, $\operatorname{PER}[\Gamma]$, to be a SINR $(\Gamma)$-dependent real-valued function with $\Gamma$ assumed Gaussian and stationary over the packet duration. Consequently, the $k$-th user SINR is:

$$
\Gamma[k]=\frac{E_{s}[k]}{N_{0}+\frac{\theta}{N} \sum_{j<k} \epsilon_{j}(\Gamma[j]) E_{s}[j]+\frac{\theta}{N} \sum_{j>k} E_{s}[j]}
$$

with $N_{0}$ the total gateway's noise power spectral density, $\frac{\theta}{N}$ the average decorrelation factor between different usersignatures in the long-code model [13], $\theta$ a decorrelation factor accounting for small inter-user timing variations [14], and $\epsilon_{j}(\Gamma[j])$ a $\Gamma$-dependent continuous random variable, independent and identically distributed among all users indicating the remaining energy fraction on the input signal after each user-cancellation.

\subsection{User-Asymptotic expressions}

In the user-asymptotic case, it is worth to define the continuous user index $t \triangleq \lim _{K \rightarrow+\infty} \frac{k}{K}$ whilst $N \rightarrow+\infty$, so that $\alpha$ remains fixed [10][11]. We also convert the discrete variables $E_{\mathrm{x}}[k], h[k], E_{s}[k], \Gamma[k]$ to the asymptotic profiles $E_{\mathrm{x}}(t), h(t)$, $E_{s}(t), \Gamma(t)$ and the corresponding summations to integrals, with $\mathrm{d} t \triangleq \lim _{K \rightarrow+\infty} \frac{1}{K}$. In this case, $\Gamma[k]$ in (2) is turned into the following asymptotic continuous profile

$$
\Gamma(t)=\frac{E_{s}(t)}{N_{0}+\alpha \theta \int_{0}^{t} \epsilon_{\tau}(\Gamma(\tau)) E_{s}(\tau) \mathrm{d} \tau+\alpha \theta \int_{t}^{1} E_{s}(\tau) \mathrm{d} \tau}
$$

Additionally, when sufficiently smooth $E_{s}(t)$ profiles at every infinitesimal interval $[t, t+\mathrm{d} t]$ are considered, the random term $\epsilon_{t}(\Gamma(t))$ can be substituted by its expected value, $\varepsilon(\Gamma(t))$, referred to as RE function ${ }^{1}$. Analogously to [15], we isolate $N_{t}(t) \triangleq E_{s}(t) / \Gamma(t)$ from (3) and we apply the differentiation operator $\nabla_{t}$ before dividing by $N_{t}(t)$ at both sides. After some manipulations, it is easy to obtain the Ordinary Differential Equation (4) that relates $\Gamma(t)$ for a known $E_{s}(t)$, and, whose analytic solution becomes (5):

$$
\begin{aligned}
\nabla_{t} \log E_{s}(t) & =\nabla_{t} \log \Gamma(t)-\alpha \Phi[\Gamma(t)] \\
E_{s}(t) & =\Gamma(t) N_{t}(0) \exp (-B(t))
\end{aligned}
$$

with $\Phi[\Gamma] \triangleq \theta(1-\varepsilon(\Gamma)) \Gamma$ a function that only depends on the FEC decoder system implementation, $N_{t}(0)=N_{0}+\theta \mathcal{C}$ the initial noise term, and $B(t) \triangleq \alpha \int_{0}^{t} \Phi[\Gamma(\tau)] \mathrm{d} \tau$.

\section{PROBLEM FORMULATION}

As stated before, the goal of this Section is focused on the derivation of the optimum ordered energy distribution that maximizes the network throughput. In terms of the spectral efficiency (SE) (6), we address the following optimization problem, which includes the sum power constraint (7) needed in order to set the OP (1):

$$
\begin{aligned}
\max _{0<t_{0} \leq 1} \max _{\Gamma(t)}[\mathrm{SE}[\mathrm{bps} / \mathrm{Hz}] & \left.\triangleq \alpha \eta \int_{0}^{t_{0}} \operatorname{PSR}[\Gamma(t)] \mathrm{d} t\right] \\
\text { s.t. } & \mathcal{C} \cdot \alpha^{-1}=\int_{0}^{t_{0}} \Gamma(t) N_{t}(t) \mathrm{d} t
\end{aligned}
$$

with $\eta$ the spectral efficiency of the coding and modulation scheme, and PSR $[\Gamma] \triangleq 1-\operatorname{PER}[\Gamma]$ the Packet Success Rate function. The previous optimization problem comprises: (i) a VC problem over a continuously differentiable $\Gamma(t)$ profile between $0 \leq t \leq t_{0}$ for the fixed end points $\left\{0, t_{0}\right\}$, and, (ii) an univariate optimization over the user index $t_{0}$, which is incorporated as a load regulation mechanism [10][11].

${ }^{1} \varepsilon(\Gamma)$ depends on the specific implementation of the soft-output FEC decoder, whereby $s[n]$ is estimated as $\hat{s}[n]$ from the noisy received signal $s[n]+w[n]$, as follows:

$$
\varepsilon(\Gamma)=\mathrm{E}\left[\sum_{n}|s[n]-\hat{s}[n]|^{2}\right] / \mathrm{E}\left[\sum_{n}|s[n]|^{2}\right] .
$$




\subsection{Solution to the constrained VC problem}

We address the prior VC problem by deriving its Stationary Point (SP) equation and Transversality Condition (TC). We define the Lagrangian $\mathcal{L}$ that contains the functional (6) and incorporates (7) using the Lagrange multiplier $\lambda$, as follows:

$$
\mathcal{L} \triangleq \int_{0}^{t_{0}} \operatorname{PSR}[\Gamma(t)] \mathrm{d} t-\lambda\left(\int_{0}^{t_{0}} \Gamma(t) N_{t}(t) \mathrm{d} t-\frac{C}{\alpha}\right) .
$$

We address the optimization in the space of functions considering variations $\Gamma(t)=\Gamma_{0}(t)+a \cdot v(t)$ over $0 \leq t \leq t_{0}$, where $\Gamma_{0}(t)$ constitutes the SP SINR profile, and, $a, v(t)$ the magnitude and direction of those variations in that space. At the SP, the limit $\lim _{a \rightarrow 0} \nabla_{a} \mathcal{L}$ must vanish for every admissible $v(t)$ :

$$
\begin{aligned}
\lim _{a \rightarrow 0} \nabla_{a} \mathcal{L} & =\int_{0}^{t_{0}} \operatorname{PSR}^{\prime}\left[\Gamma_{0}(t)\right] v(t) \mathrm{d} t-\lambda \int_{0}^{t_{0}} N_{t}(t) v(t) \mathrm{d} t \\
& +\alpha \lambda \int_{0}^{t_{0}} E_{s}(t)\left(\int_{0}^{t} \Phi^{\prime}\left[\Gamma_{0}(\tau)\right] v(\tau) \mathrm{d} \tau\right) \mathrm{d} t . \quad(9)
\end{aligned}
$$

Integrating the last term by parts with $u \triangleq \int_{0}^{t} \Phi^{\prime}\left[\Gamma_{0}(\tau)\right] v(\tau) \mathrm{d} \tau$ and $d v \triangleq E_{s}(t) \mathrm{d} t$, we obtain $\int_{0}^{t_{0}} I(t) \Phi^{\prime}\left[\Gamma_{0}(t)\right] v(t) \mathrm{d} t$ where $I(t) \triangleq \int_{t}^{t_{0}} E_{s}(\tau) \mathrm{d} \tau$. Hence, (9) is re-expressed as follows

$$
\int_{0}^{t_{0}}\left[\operatorname{PSR}^{\prime}\left[\Gamma_{0}(t)\right]-\lambda\left(N_{t}(t)-\alpha I(t) \Phi^{\prime}\left[\Gamma_{0}(t)\right]\right)\right] v(t) \mathrm{d} t
$$

which must be set to zero for every admissible $v(t)$. Using the Fundamental Lemma of the Calculus of Variations [16], this reduces to setting the term in brackets to zero. Hence, the following Invariance Equation (IE) in $0 \leq t \leq t_{0}$ is obtained:

$$
\lambda=\frac{\operatorname{PSR}^{\prime}\left[\Gamma_{0}(t)\right]}{N_{t}(t)-\alpha I(t) \Phi^{\prime}\left[\Gamma_{0}(t)\right]}
$$

with $\lambda>0$ used to set the constraint (7), and computed by evaluating (10) at $t=0$ with the boundary $\alpha I(0)=\mathcal{C}$, which gives $\lambda=\operatorname{PSR}^{\prime}\left[\Gamma_{0}(0)\right] /\left(N_{0}+\mathcal{C} \theta-\mathcal{C} \Phi^{\prime}\left[\Gamma_{0}(0)\right]\right)$. The obtained solution inherits the properties derived in [10], in which the optimum SINR and energy profiles at the SIC input are shown to be non-increasing functions of the variable $t$.

\subsubsection{Derivation of the TC}

To obtain the TC corresponding to the optimum $t_{0}$, we set the derivative of (8) w.r.t. $t_{0}$ to zero, getting $\operatorname{PSR}\left[\Gamma_{0}\left(t_{0}\right)\right]=$ $\lambda \Gamma_{0}\left(t_{0}\right) N_{t}\left(t_{0}\right)$. Then, evaluating (10) at $t=t_{0}$, we obtain $\operatorname{PSR}^{\prime}\left[\Gamma_{0}\left(t_{0}\right)\right]=\lambda N_{t}\left(t_{0}\right)$. Combining both equations, we get

$$
\operatorname{PSR}\left[\Gamma_{0}\left(t_{0}\right)\right]=\operatorname{PSR}^{\prime}\left[\Gamma_{0}\left(t_{0}\right)\right] \Gamma_{0}\left(t_{0}\right),
$$

which is referred to as TC, stating that, under network overload, load regulation $t_{0}<1$ is enabled so that the SINR value of the user $t_{0}$ is mapped onto a specific point of the FEC decoder characteristic $\operatorname{PSR}^{\prime}\left[\Gamma^{*}\right] \Gamma^{*}=\operatorname{PSR}\left[\Gamma^{*}\right]$. This point is also related to the elasticity of the $\operatorname{PSR}[\Gamma]$ function: $\mathrm{e}\left(\Gamma^{*}\right) \triangleq \mathrm{d} \log (\operatorname{PSR}[\Gamma]) /\left.\mathrm{d} \log (\Gamma)\right|_{\Gamma=\Gamma^{*}}=1$. This highlights that user (traffic) admission is performed until small last-user log-SINR changes result in identical log-PSR variations.

As stated in [10], $\Gamma_{0}(t)>\Gamma_{\text {ip }}$ in $\left[0, t_{0}\right]$ is a sufficient condition that guarantees $\Gamma_{0}(t)$ being a local-maximizer of the SE functional (6), with $\Gamma_{\text {ip }} \triangleq \operatorname{PSR}^{\prime \prime-1}[0]$ (the unique code inflexion point). In our case, since $\Gamma_{0}(t) \geq \Gamma^{*}$, we recall the mean value theorem with $f(\Gamma) \triangleq \operatorname{PSR}^{\prime}[\Gamma] \Gamma-\operatorname{PSR}[\Gamma]$ in $\left[0, \Gamma^{*}\right]$. As $f(0)=f\left(\Gamma^{*}\right)=0, \Gamma_{+} \in\left(0, \Gamma^{*}\right)$ exists such that $f^{\prime}\left(\Gamma_{+}\right)=\operatorname{PSR}^{\prime \prime}\left[\Gamma_{+}\right] \Gamma_{+}=0$. This implies $\Gamma_{+}=\Gamma_{\text {ip }}<\Gamma^{*}$.

\section{OPTIMUM WORKING POINT}

In this Section, we analyze the maximum achievable network throughput when load regulation, clarified in Section 3.1.1, occurs. First, we turn the IE (10) into an exact differential, and finally, we characterize that maximum point.

We take the IE in (10) and we re-express it by setting: $\operatorname{PSR}^{\prime}[\Gamma]=\mathrm{dPSR}[\Gamma] / \mathrm{d} \Gamma$, and $\Phi^{\prime}[\Gamma]=\mathrm{d} \Phi[\Gamma] / \mathrm{d} \Gamma$, as (12). Using $N_{t} \mathrm{~d} \Gamma=\mathrm{d}\left(\Gamma N_{t}\right)-\Gamma \mathrm{d} N_{t}$ and $\mathrm{d} N_{t}=-\alpha \Phi[\Gamma] N_{t} \mathrm{~d} t=$ $\alpha \mathrm{d}(I \Phi[\Gamma]) / \Gamma$, we convert it into the exact differential (13)

$$
\begin{aligned}
& \mathrm{dPSR}\left[\Gamma_{0}(t)\right]=\lambda\left(N_{t}(t) \mathrm{d} \Gamma_{0}(t)-\alpha I(t) \mathrm{d} \Phi\left[\Gamma_{0}(t)\right]\right) \\
& \mathrm{dPSR}\left[\Gamma_{0}(t)\right]=\lambda \cdot \mathrm{d}\left(E_{s}(t)-\alpha I(t) \Phi\left[\Gamma_{0}(t)\right]\right)
\end{aligned}
$$

which highlights that power allocation is done in such a way that increments of the user's success $\operatorname{PSR}[\Gamma]$ are proportional to increments over the right-hand side magnitude in (13). Hence, integrating at both sides between $[0, t]$, we obtain an equation alternative to (10) to compute $\Gamma_{0}(t)$ in $0 \leq t \leq t_{0}$ :

$$
\lambda=\frac{\operatorname{PSR}\left[\Gamma_{0}(t)\right]-\operatorname{PSR}\left[\Gamma_{0}(0)\right]}{E_{s}(t)-E_{s}(0)-\alpha I(t) \Phi\left[\Gamma_{0}(t)\right]+\mathcal{C} \Phi\left[\Gamma_{0}(0)\right]} .
$$

The rest of the section is focused on the load regulated state. Evaluating (14) at $t=t_{0}$ with $I\left(t_{0}\right)=0$, making use of $\operatorname{PSR}\left[\Gamma_{0}\left(t_{0}\right)\right]=\lambda E_{s}\left(t_{0}\right)$ (see Section 3.1.1), and simplifying terms, we obtain the right hand side equation, which results equivalent to the following

$$
\lambda=\frac{\operatorname{PSR}^{\prime}\left[\Gamma_{0}(0)\right]}{N_{t}(0)-\mathcal{C} \Phi^{\prime}\left[\Gamma_{0}(0)\right]}=\frac{\operatorname{PSR}\left[\Gamma_{0}(0)\right]}{\Gamma_{0}(0) N_{t}(0)-\mathcal{C} \Phi\left[\Gamma_{0}(0)\right]}
$$

which constitutes a non-linear equation in $\Gamma_{0}(0)=\Gamma_{0}^{*}$, that, together with $\Gamma^{*}$, also remains fixed when $t_{0}<1$ is enabled. Therefore, making use of (15), (14) reduces to the following

$$
\operatorname{PSR}\left[\Gamma_{0}(t)\right]=\lambda\left(E_{s}(t)-\alpha I(t) \Phi\left[\Gamma_{0}(t)\right]\right) .
$$

We are interested in evaluating the SE magnitude when load regulation occurs. For that reason, we have computed the rate of change of the network SE as a function of the traffic load. Specifically, using (5), the derivative of $\alpha \eta \mathcal{L}$ w.r.t. $\alpha$ is

$$
\eta \int_{0}^{t_{0}} \operatorname{PSR}[\Gamma(t)] \mathrm{d} t-\frac{\lambda \eta C}{\alpha}+\lambda \eta \alpha \int_{0}^{t_{0}} I(t) \Phi[\Gamma(t)] \mathrm{d} t .
$$

Making use of (16) and (7), this gives $\mathrm{dSE} / \mathrm{d} \alpha=0$. Hence, when load regulation $t_{0}<1$ acts, the network SE saturates so that only a maximum traffic of $\alpha t_{0}$ can be served. 


\section{SIMULATIONS}

We have evaluated the user-asymptotic expressions derived herein for a spread-spectrum multiple access scenario of QPSK-modulated users sharing the standardised DVB-RCS Turbo Code (TC) of rate $1 / 2(\eta=1)$ with a packet length of 440 information bits [17]. As the network performance may depend on the adopted FEC code, we have considered for the same encoder, the case of performing 1 (TC1) or 5 (TC2) Max-log-map iterations. Both the PER $[\Gamma]$ and the $\varepsilon(\Gamma)$ curves of the said FEC decoders are depicted in Fig. 1.

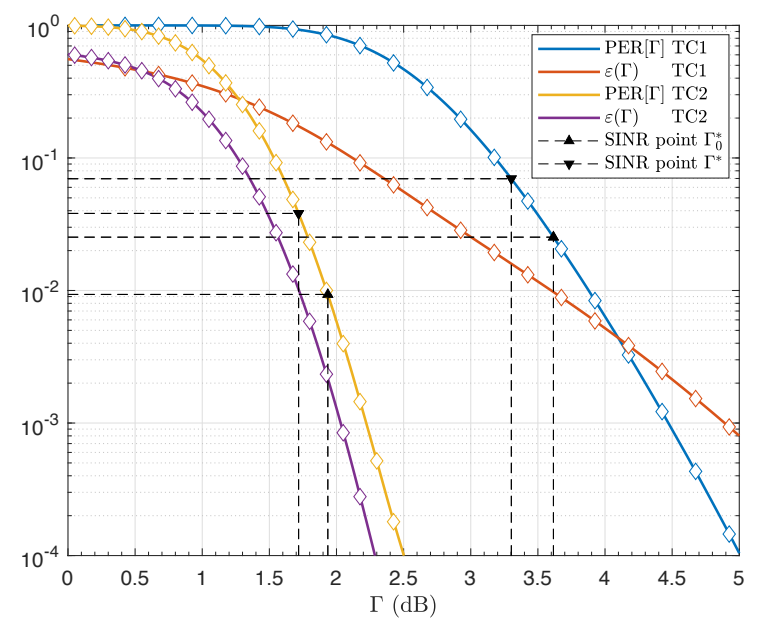

Fig. 1. $\operatorname{PER}[\Gamma]$ and $\varepsilon(\Gamma)$ vs. $\Gamma$ curves. Diamond markers denote the simulated Monte Carlo points whereas solid lines indicate the interpolated regions. Dashed lines show the PER values associated to $\Gamma_{0}^{*}$ and $\Gamma^{*}$ for the soft SIC case.

For simulation purposes, we set $\mathcal{C} / N_{0}=10 \mathrm{~dB}$, the decorrelation factor $\theta$ to 1 , and, for simplicity, we fix $G_{s} h_{d}=1$. Fig. 2 depicts, for both FEC decoders, the network SE as a function of the system's traffic load $\alpha$. In order to evidence the benefit of the adopted soft SIC, we have also simulated the hard SIC [10], for which, after Cyclic Redundancy Check (CRC), only those successfully decoded packets are reconstructed and cancelled from the input signal. As shown in Fig. 2, soft SIC does not benefit in terms of raw throughput when the system is underloaded, whilst a relevant gain is achieved for higher traffic activity situations at the same receiver decoding-complexity cost. When network overload occurs, the system's SE saturates, and that point has been found to exactly coincide with the load regulation activation $t_{0}<1$, which validates the derivation at the end of Section 4 . In particular, the saturation load of both TC1 and TC2 codes is found to be at 1.06 and 1.56 , with values 1.00 and 1.53 bps/Hz, respectively. For the same FEC decoders, the adopted policy in [10] presents saturation loads of 0.99 and 1.51 with respective SE values of 0.96 and $1.49 \mathrm{bps} / \mathrm{Hz}$. Their individual net SE gains exclude the hard SIC's adopted CRC length.
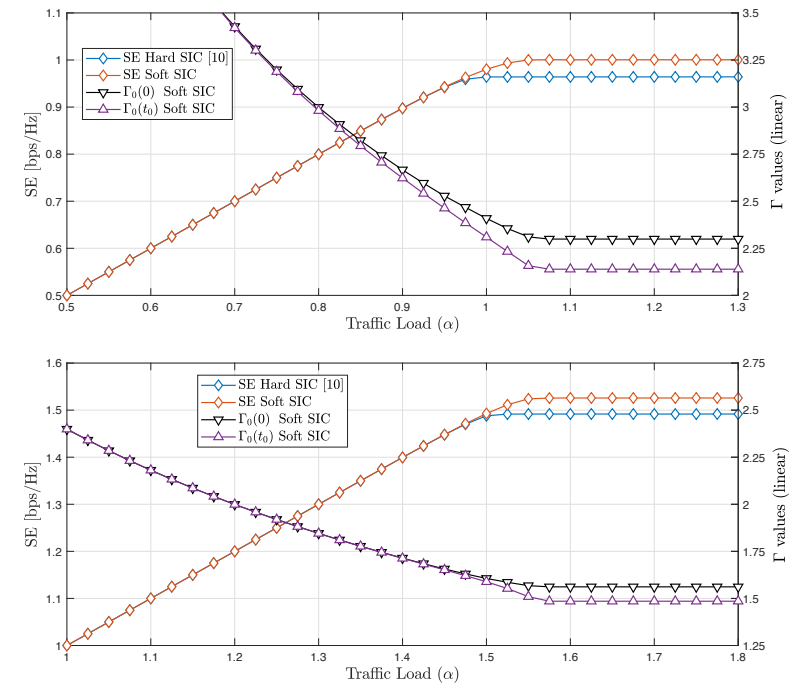

Fig. 2. Spectral efficiency and SINR values $\Gamma_{0}(0)$ and $\Gamma_{0}\left(t_{0}\right)$ vs. traffic load $\alpha$ for the TC1 (above) and TC2 (below) codes.

Hence, for the case of 8 and 16 bits, we achieve SE improvements of $5.7 \%$ and $7.7 \%$ for the TC1 and $4.2 \%$ and $6.2 \%$ for the $\mathrm{TC} 2$. In all cases, as zero CRC error detection is assumed, the prior gains are in fact lower bounds for the network SE.

For the case study, we have also depicted the maximum $\Gamma_{0}(0)$ and minimum $\Gamma_{0}\left(t_{0}\right)$ values of $\Gamma_{0}(t)$ at each traffic load. In underloaded network situations, $\Gamma_{0}(t)$ is practically uniform while in high traffic activity cases moderate discrepancies appear, and, whose magnitude depend on how abrupt $\operatorname{PER}[\Gamma]$ and $\varepsilon(\Gamma)$ are. This conclusion is also supported by simulations carried in [10], and contrasts with the fact that the maximum sum-rate capacity SINR profile is uniform over consecutive SIC stages [18]. Finally, in overloaded circumstances $\Gamma_{0}(0), \Gamma_{0}\left(t_{0}\right)$ become the SINR values $\Gamma_{0}^{*}, \Gamma^{*}$, where $t_{0} \leq 1$ regulates the admitted load so that $\alpha t_{0}$ remains fixed.

\section{CONCLUSIONS}

We have shown, for a Successive Soft Interference Cancellation scheme operating in the large-user regime, that VC can be successfully used to derive the optimum user-energy profile that maximizes the network spectral efficiency when the sum power at the SIC input is constrained. In that respect, we have derived the Stationary Point equation and the associated Transversality Condition of the stated VC problem. We have proved that under high traffic activity, the network may regulate the admitted load in order to set a specific operating point for the first and the last user in the SIC decoding order, so that the network throughput saturates. We evidence that the throughput gain achieved using soft SIC in view of the traditional SIC policies is increased for smoother PER curves. We also show the convenience of soft SIC under high traffic activity, with emphasis in short packet length services, where reducing user payload overheads becomes a critical factor. 


\section{REFERENCES}

[1] Z. Dawy, W. Saad, A. Ghosh, J. G. Andrews, and E. Yaacoub, "Toward massive machine type cellular communications," IEEE Wireless Communications, vol. 24, no. 1, pp. 120-128, February 2017.

[2] F. Collard and R. De Gaudenzi, "On the optimum packet power distribution for spread aloha packet detectors with iterative successive interference cancelation," IEEE Transactions on Wireless Communications, vol. 13, no. 12, pp. 6783-6794, December 2014.

[3] ETSI EN, "Digital Video Broadcasting (DVB); Second Generation DVB Interactive Satellite System (DVBRCS2); Part 2: Lower Layers for Satellite standard," ETSI EN 301 545-2 V1.1.1, January 2012.

[4] ETSI TS, "Satellite Earth Stations and Systems; Air Interface for S-band Mobile Interactive Multimedia (SMIM)," ETSI TS 102 721, December 2011.

[5] R. De Gaudenzi and O. del Rio Herrero, "Advances in random access protocols for satellite networks," in International Workshop on Satellite and Space Communications, September 2009, pp. 331-336.

[6] G. Gallinaro, N. Alagha, R. De Gaudenzi, K. Kansanen, R. Müller, and P. Salvo Rossi, "ME-SSA: An advanced random access for the satellite return channel," in 2015 IEEE International Conference on Communications (ICC), June 2015, pp. 856-861.

[7] A. Reichman, "Enhanced Spread Spectrum Aloha (E-SSA), an emerging satellite return link messaging scheme," in IEEE 28th Convention of Electrical Electronics Engineers in Israel (IEEEI), December 2014, pp. $1-4$.

[8] O. Del Rio Herrero and R. De Gaudenzi, "High efficiency satellite multiple access scheme for machineto-machine communications," IEEE Transactions on Aerospace and Electronic Systems, vol. 48, no. 4, pp. 2961-2989, October 2012.

[9] P. Salvo Rossi, K. Kansanen, R. R. Müller, and C. Rachinger, "Power randomization for iterative detection over random-access fading channels," IEEE Transactions on Wireless Communications, vol. 14, no. 10, pp. 5704-5713, October 2015.

[10] J. Sala-Alvarez, F. Rey Micolau, J. Villares, and F. Molina, "Minimum PER user-energy profile for massive SIC receivers under an average energy constraint," in IEEE International Workshop on Signal Processing Advances for Wireless Communications, 2017 July, pp. $700-705$.
[11] J. Villares, F. Rey Micolau, and J. Sala-Alvarez, "Optimum energy allocation for massive spread-spectrum multiple access in networks of uncoordinated energylimited terminals," in IEEE 17th International Workshop on Signal Processing Advances in Wireless Communications (SPAWC), July 2016, pp. 1-6.

[12] F. Lázaro, "Decentralized power control for slotted spread spectrum aloha with successive interference cancellation," in SCC 2017; 11th International ITG Conference on Systems, Communications and Coding; Proceedings of. VDE, 2017, pp. 1-6.

[13] S. Verdu and S. Shamai, "Spectral efficiency of CDMA with random spreading," IEEE Transactions on Information Theory, vol. 45, no. 2, pp. 622-640, March 1999.

[14] J. Sala-Alvarez, J. Villares, and F. Rey Micolau, "Asymptotic and finite user PER analysis of successive interference cancellation for DS-CDMA," IEEE Communications Letters, vol. 15, no. 11, pp. 1145-1147, November 2011.

[15] J. Sala-Alvarez, F. Rey Micolau, and J. Villares, "Asymptotically optimum energy profile for successive interference cancellation in DS-CDMA under a power unbalance constraint," IEEE Communications Letters, vol. 16, no. 2, pp. 172-175, February 2012.

[16] Izrail Moiseevitch Gelfand, Richard A Silverman, et al., Calculus of Variations, Courier Corporation, 2000.

[17] ETSI EN, "Digital Video Broadcasting (DVB); Interaction channel for satellite distribution systems," ETSI EN 301790 V1.5.1, May 2005.

[18] D. V. Djonin and V. K. Bhargava, "Asymptotic analysis of the conventional decision feedback receiver in fading channels," IEEE Transactions on Wireless Communications, vol. 2, no. 5, pp. 1066-1078, September 2003. 